\title{
Patient Navigation Program
}

National Cancer Institute

\section{Source}

National Cancer Institute. Patient Navigation Program. NCI Thesaurus. Code C139294.

A healthcare program that is designed to guide a patient through the healthcare system and reduce barriers to timely screening, follow-up, diagnosis, treatment, and supportive care. 